International Mathematical Forum, Vol. 9, 2014, no. 22, 1053 - 1059

HIKARI Ltd, www.m-hikari.com

http://dx.doi.org/10.12988/imf.2014.4472

\title{
Convergence Theorems for a Finite Family of Relatively Quasi-Nonexpansive Mappings and System of Equilibrium Problems
}

\author{
B. G. Akuchu \\ Department of Mathematics \\ University of Nigeria \\ Nsukka, Nigeria
}

Copyright (c) 2014 B. G. Akuchu. This is an open access article distributed under the Creative Commons Attribution License, which permits unrestricted use, distribution, and reproduction in any medium, provided the original work is properly cited.

\begin{abstract}
In this paper, we employ an iteration scheme introduced in [13], to prove strong convergence to a common element of the set of fixed points of a family $\left\{T_{i}\right\}_{i=1}^{N}$ of $L_{i}$-Lipschitzian mappings and the set of common solutions to a system of equilibrium problems, in a uniformly convex Banach space which is also uniformly smooth. We do not require that the family $\left\{T_{i}\right\}_{i=1}^{N}$ be uniformly continuous, as in [13].
\end{abstract}

\section{Introduction}

Let $E$ be a real Banach space and let $C$ be a nonempty subset of $E$. A mapping $T: C \rightarrow C$ is called Lipschitzian, if there exists $L>0$ such that

$$
\|T x-T y\| \leq L\|x-y\|,
$$

for all $x, y \in C$. If $L<1$, then $T$ is called a contraction. If $L=1$, then $T$ is called nonexpansive.

A point $x \in C$ is called a fixed point of $T$ if $T x=x$. The set of fixed points of $T$ is defined as $F(T):=\{x \in C: T x=x\}$. If $F(T) \neq \emptyset$ and $T$ satisfies

$$
\|T x-p\| \leq\|x-p\|
$$

for all $x \in C, p \in F(T)$, then $T$ is called Quasi-Nonexpansive. Observe that every nonepansive mapping with a nonempty fixed point set is a quasinonexpansive mapping. 
Let $E^{E^{*}}$, be the dual space of $E$, defined by

$$
J(x)=\left\{f \in E^{*}:\langle x, f\rangle=\|x\|^{2}=\|f\|^{*}\right\} .
$$

If $E$ is smooth, then $J$ is single-valued.

Throughout this paper, we denote by $\phi$, the functional on $E \times E$ defined by

$$
\phi(x, y):=\|x\|^{2}-2\langle x, j(y)\rangle+\|y\|^{2}
$$

for all $x, y \in C$.

A point $y \in C$ is said to be an asymptotic fixed point of $T$, if $C$ contains a sequence $\left\{x_{n}\right\}$ which converges weakly to $y$ and $\lim \left\|x_{n}-T x_{n}\right\|=0$. The set of asymptotic fixed points of $T$ is denoted by $\bar{F}$. We say that a mapping $T$ is relatively nonexpansive (see for example [3-5] and the references therein) if the following conditions are satisfied:

$\left(R_{1}\right) F(T) \neq \emptyset$

$\left(R_{2}\right) \phi(p, T x) \leq \phi(p, x), \forall x \in C, p \in F(T)$

$\left(R_{3}\right) F(T)=\bar{F}(T)$

If $T$ satisfies $R_{1}$ and $R_{2}$, then $T$ is said to be relatively quasi-nonexpansive. It is easily seen that the class of relatively quasi-nonexpansive mappings contains the class of relatively nonexpansive maps. Several authors (see for example [9], [10] and the references therein) have studied the approximations of fixed points of relatively quasi-nonexpansive mappings. It is easy to see that in a Hilbert space, $H$, the classes of relatively quasi-nonexpansive and relatively nonexpansive mappings coincide. This is because $\phi(x, y)=\|x-y\|^{2}, \forall x, y \in H$ and this implies $\phi(p, T x) \leq \phi(p, x) \Leftrightarrow\|T x-p\| \leq\|x-p\| \forall x \in C, p \in F(T)$. Examples of relatively quasi-nonexpansive mappings are given in [10].

Let $F$ be a bi-function of $C \times C \rightarrow \Re$. The equilibrium problem is to find $x \in C$ such that

$$
F(x, y) \geq 0
$$

for all $y \in C$. The set of solutions to (1.1) is denoted by $\operatorname{EP}(F):=\left\{x^{*} \in\right.$ $\left.C: F\left(x^{*}, y\right) \geq 0 \forall y \in C\right\}$. Many problems in Engineering, Economics and optimization are reduced to finding a solution of (1.1). Several methods have been proposed for solving (1.1) (see for example [2], [6] and the references therein).

Takahashi and Zembayashi [12] introduced a hybrid iterative scheme for the approximation of fixed points of relatively nonexpansive mappings which are also solutions to equilibrium problems, in a uniformly smooth real Banach space which is also uniformly convex.

Recently, Yekini [13] proved strong convergence theorems for a finite family of relatively quasi-nonexpansive mappings and a system of equilibrium problems in a real uniformly convex Banach space which is also uniformly smooth. More precisely, he proved the following theorem: 
Theorem 1 [13]: Let $E$ be a real uniformly convex Banach space which is also uniformly smooth. Let $C$ be a nonempty closed convex subset of $E$. For each $k=1,2, \ldots, m$ let $F_{k}$ be a function from $C \times C \rightarrow \Re$, satisfying $\left(A_{1}\right)-\left(A_{4}\right)$ and let $\left\{T_{i}\right\}_{i=1}^{N}$ be a finite family of closed relatively quasi-nonexpansive mappings of $C$ into itself such that $F:=\cap_{i=1}^{N} F\left(T_{i}\right) \cap\left(\cap_{k=1}^{m} E P\left(F_{k}\right)\right) \neq \emptyset$. Assume that $T_{i}$ is uniformly continuous for each $i=1,2,3, \ldots, N$. Let $\left\{x_{n}\right\}$ be iteratively generated by $x_{0} \in C, C_{1}=C, x_{1}=\Pi_{C_{1}} x_{0}$,

$$
\left\{\begin{array}{l}
y_{n}=J^{-1}\left(\alpha_{n} J x_{n}+\left(1-\alpha_{n}\right) J T_{n} x_{n}\right), n \geq 1 \\
u_{n}=T_{r_{m}, n}^{F_{m}} T_{r_{m-1, n}}^{F_{m-1}} \ldots T_{r_{2, n}}^{F_{2}} T_{r_{1, n}}^{F_{1}} y_{n} \\
C_{n+1}=\left\{w \in C_{n}: \phi\left(w, u_{n}\right) \leq \phi\left(w, x_{n}\right), n \geq 1\right. \\
x_{n+1}=\prod_{C_{n+1}} x_{0}, n \geq 1
\end{array}\right.
$$

where $J$ is the duality mapping on $E$ and $T_{n}:=T_{n(\bmod N)}$. Suppose $\left\{\alpha_{n}\right\}$ is a sequence in $(0,1)$ such that $\lim \inf \alpha_{n}\left(1-\alpha_{n}\right)>0$ and $\left\{r_{k, n}\right\},(k=1,2, \ldots, m)$ satisfying $\liminf r_{k, n}>0,(k=1,2, \ldots, m)$. Then $\left\{x_{n}\right\}$ converges strongly to $\Pi_{F} x_{0}$.

Motivated by the above theorem, we prove strong convergence theorems for a finite family $\left\{T_{i}\right\}_{i=1}^{N}$ of $L_{i}$-Lipschitzian relatively quasi-nonexpansive mappings and a system of equilibrium problems in a uniformly convex Banach space which is also uniformly smooth. Our finite family $\left\{T_{i}\right\}_{i=1}^{N}$ is different from that in [13], as ours is a family of $L_{i}$-Lipschitzian mappings as opposed to the uniform continuity assumed for the family $\left\{T_{i}\right\}_{i=1}^{N}$ in [13].

\section{Preliminaries}

Let $E$ be a real Banach space. The modulus of smoothness of $E$ is the function $\rho_{E}:[0, \infty) \rightarrow[0, \infty)$ defined by

$$
\rho_{E}(\tau):=\left\{\frac{1}{2}(\|x+y\|+\|x-y\|)-1:\|x\| \leq 1,\|y\| \leq \tau\right\} .
$$

$E$ is uniformly smooth if and only if $\lim _{\tau \rightarrow 0} \frac{\rho_{E}(\tau)}{\tau}=0$.

The modulus of convexity of $E$ is the function $\delta_{E}:(0,2] \rightarrow[0,1]$ defined by

$$
\delta_{E}(\epsilon):=\inf \left\{1-\left\|\frac{x+y}{2}\right\|:\|x\|=\|y\|=1 ; \epsilon=\|x-y\| .\right.
$$

$E$ is said to be uniformly convex if for any $\epsilon \in(0,2]$, there exists a $\delta=$ $\delta(\epsilon)>0$ such that if $x, y \in E$ with $\|x\| \leq 1,\|y\| \leq 1$ and $\|x-y\| \geq \epsilon$, then $\left\|\frac{1}{2}(x+y)\right\| \leq 1-\delta$. Equivalently, $E$ is uniformly convex if and only if $\delta_{E}(\epsilon)>0$ for all $\epsilon \in(0,2]$. A normed space is called strictly convex if for all $x, y \in E, x \neq y,\|x\|=\|y\|=1$, we have $\|\lambda x+(1-\lambda) y\|<1, \forall \lambda \in(0,1)$.

Let $E$ be a smooth, strictly convex and reflexive Banach space and $C$ be a nonempty closed convex subset of $E$. Then the generalized projection $\Pi_{C}$ from 
$E$ onto $C$ is defined by

$$
\Pi_{C}(x):=\operatorname{argmin}_{y \in C} \phi(x, y), \forall x \in E .
$$

The existence and uniqueness of $\Pi_{C}$ follows from the property of the functional $\phi(x, y)$ and the strict monotonicity of the mapping $J$ (see for example [1]). If $E$ is a Hilbert space, then $\Pi_{C}$ is the metric projection from $H$ onto $C$. It is well known (see for example [7]), that in uniformly convex and uniformly smooth Banach spaces

$$
(\|x\|-\|y\|)^{2} \leq \phi(x, y) \leq(\|x\|+\|y\|)^{2}, \forall x, y \in E .
$$

We now state some well known lemmas, which will be useful in the sequel.

Lemma 1 [1]: Let $C$ be a nonempty closed convex subset of a smooth, strictly convex and reflexive Banach space, $E$. Then

$$
\phi\left(x, \Pi_{C} y\right)+\phi\left(\Pi_{C} y, y\right) \leq \phi(x, y), \forall x \in C, \forall y \in E
$$

Lemma 2[1]: Let $C$ be a nonempty closed convex subset of a smooth, strictly convex and reflexive Banach space, $E$. Let $x \in E$ and $z \in C$. Then

$$
z=\Pi_{C} x \Leftrightarrow\langle y-z, J(x)-J(z)\rangle \leq 0, \forall y \in C
$$

Lemma 3[10]: Let $T$ be a closed relatively quasi-nonexpansive mapping of $C$ into itself. Then $F(T)$ is closed and convex.

Lemma 4 [7]: Let $C$ be a nonempty closed convex subset of a smooth, strictly convex and reflexive Banach space, $E$. Let $\left\{x_{n}\right\}$ and $\left\{y_{n}\right\}$ be sequences in $E$ such that either $\left\{x_{n}\right\}$ or $\left\{y_{n}\right\}$ is bounded. If $\lim _{n \rightarrow \infty} \phi\left(x_{n}, y_{n}\right)=0$, then $\lim _{n \rightarrow \infty}\left\|x_{n}-y_{n}\right\|=0$.

Lemma 5 [14]: Let $E$ be a real uniformly convex Banach space. For arbitrary $r>0$, let $B_{r}(0):=\{x \in E:\|x\| \leq r\}$ and $\lambda \in[0,1]$. Then there is a continuous strictly increasing convex function $g:[0,2 r] \rightarrow \Re, g(0)=0$ such that for every $x, y \in B_{r}(0)$, the following inequality holds:

$$
\|\lambda x+(1-\lambda) y\|^{2} \leq \lambda\|x\|^{2}+(1-\lambda)\|y\|^{2}-\lambda(1-\lambda) g\left(\|x-y\|^{2}\right)
$$

For solving the equilibrium problem for a bifunction $F: C \times C \rightarrow \Re$, we assume $F$ satisfies the following conditions:

$\left(A_{1}\right) F(x, x)=0 \forall x \in C$

$\left(A_{2}\right)$ Fismonotone, i.eF $(x, y)+F(y, x) \leq 0 \forall x, y \in C$

$\left(A_{3}\right)$ foreach, $x, y \in C, \lim _{t \rightarrow 0} F(t z+(1-t) x, y) \leq F(x, y)$

$\left(A_{4}\right)$ foreach $x \in C, y \mapsto F(x, y)$ is convex and lower semicontinuous.

In [2], the authors proved the following lemma:

Lemma 6 [2]: Let $C$ be a nonempty closed convex subset of a smooth, strictly convex and reflexive Banach space, $E$, and let $F$ be a bifunction of $C \times C$ into 
$\Re$ satisfying $\left(A_{1}\right)-\left(A_{4}\right)$. Let $r>0$ and $x \in E$. Then there exists $z \in C$ such that

$$
F(z, y)+\frac{1}{r}\langle y-z, J z-J x\rangle \geq 0
$$

for all $y \in C$.

Takahashi and Zembayashi [11], proved the following lemma:

Lemma 7 [11]: Let $C$ be a nonempty closed convex subset of a smooth, strictly convex and reflexive Banach space, $E$. Assume that $F: C \times C \rightarrow \Re$ satisfies $\left(A_{1}\right)-\left(A_{4}\right)$. For $r>0$ and $x \in E$, define a mapping $T_{r}^{F}: E \rightarrow C$ as follows:

$$
T_{r}^{F}(x)=\left\{z \in C: F(z, y)+\frac{1}{r}\langle y-z, J z-J x\rangle \geq 0, \forall y \in C\right\},
$$

for all $z \in E$. Then, the following hold:

1. $T_{r}^{F}$ is single - valued

2. $T_{r}^{F}$ is a firmly nonexpansive-type mapping, i.e. for all $x, y \in E$,

$$
\left\langle T_{r}^{F} x-T_{r}^{F} y, J T_{r}^{F} x-J T_{r}^{F} y\right\rangle \leq\left\langle T_{r}^{F} x-T_{r}^{F} y, J x-J y\right\rangle
$$

3. $F\left(T_{r}^{F}\right)=E P(F)$

4. $E P(F)$ is closed and convex

Main Results

Theorem 2: Let $E$ be a real uniformly convex Banach space which is also uniformly smooth. Let $C$ be a nonempty closed convex subset of $E$. For each $k=1,2, \ldots, m$ let $F_{k}$ be a function from $C \times C \rightarrow \Re$, satisfying $\left(A_{1}\right)-\left(A_{4}\right)$ and let $\left\{T_{i}\right\}_{i=1}^{N}$ be a finite family of closed relatively quasi-nonexpansive mappings of $C$ into itself such that $F:=\cap_{i=1}^{N} F\left(T_{i}\right) \cap\left(\cap_{k=1}^{m} E P\left(F_{k}\right)\right) \neq \emptyset$. Assume that $T_{i}$ is $L_{i}$-Lipschitzian for each $i=1,2,3, \ldots, N$. Let $\left\{x_{n}\right\}$ be iteratively generated by $x_{0} \in C, C_{1}=C, x_{1}=\Pi_{C_{1}} x_{0}$,

$$
\left\{\begin{array}{l}
y_{n}=J^{-1}\left(\alpha_{n} J x_{n}+\left(1-\alpha_{n}\right) J T_{n} x_{n}\right), n \geq 1 \\
u_{n}=T_{r_{m}, n}^{F_{m}} T_{r_{m-1}-n}^{F_{m-1}} \ldots T_{r_{2, n}}^{F_{2}} T_{r_{1, n}}^{F_{1}} y_{n} \\
C_{n+1}=\left\{w \in C_{n}: \phi\left(w, u_{n}\right) \leq \phi\left(w, x_{n}\right), n \geq 1\right. \\
x_{n+1}=\prod_{C_{n+1}} x_{0}, n \geq 1
\end{array}\right.
$$

where $J$ is the duality mapping on $E$ and $T_{n}:=T_{n(\bmod N)}$. Suppose $\left\{\alpha_{n}\right\}$ is a sequence in $(0,1)$ such that $\liminf \alpha_{n}\left(1-\alpha_{n}\right)>0$ and $\left\{r_{k, n}\right\},(k=1,2, \ldots, m)$ satisfying $\lim \inf r_{k, n}>0,(k=1,2, \ldots, m)$. Then $\left\{x_{n}\right\}$ converges strongly to $\Pi_{F} x_{0}$.

Proof: The facts that $C_{n}$ is closed and convex, $\left\{x_{n}\right\}$ is a Cauchy sequence in $C, \lim _{n \rightarrow \infty}\left\|x_{n+l}-x_{n}\right\|=0 \forall l=1,2, \ldots, N ; \lim \left\|x_{n}-T_{n} x_{n}\right\|=0$ all follow from the proof of theorem 3.1 of [13]. 
Now since $T_{i}$ is $L_{i}$-Lipschitzian for each $i=1,2, \ldots, N$ set $L=\max \left\{L_{i}\right\}$. Then $\left\|T_{i} x-T_{i} y\right\| \leq L\|x-y\|$. Now

$$
\begin{aligned}
\left\|x_{n}-T_{n+l} x_{n}\right\| & \leq\left\|x_{n}-x_{n+l}\right\|+\left\|x_{n+l}-T_{n+l} x_{n+l}\right\|+\left\|T_{n+l} x_{n+l}-T_{n+l} x_{n}\right\| \\
& \leq\left\|x_{n}-x_{n+l}\right\|+\left\|x_{n+l}-T_{n+l} x_{n+l}\right\|+L\left\|x_{n}-x_{n+l}\right\| \\
& =(1+L)\left\|x_{n}-x_{n+l}\right\|+\left\|x_{n+l}-T_{n+l} x_{n+l}\right\| \rightarrow 0 \text { as } n \rightarrow \infty
\end{aligned}
$$

The rest of the proof now follows as from (3.7) of [13].

Remark: The classes of lipschitzian and uniformly continuous maps are independent of each other. Hence our results complement the results in [13].

\section{References}

1. Alber, Y. I., Metric and generalized projection operator in Banach spaces: properties and applications. In: Theory and Applications of Nonlinear Operators of Accretive and Monotone Type vol. 178 of Lecture Notes in Pure and Applied Mathematics, pp. 15-20. Dekker, New York (1996).

2. Blum E., Oettli, W., From optimization and variational inequalities to equilibrium problems, Math. Stud. 63, 123-145 (1994)

3. Butnariu, D., Reich, S., Zaslavski, A. J., Asymptotic behaviour of relatively nonexpansive operators in Banach spaces, J. Appl. Anal. 7, 151-174 (2001)

4. Butnariu, D., Reich, S., Zaslavski, A. J.,Weak convergence of orbits of nonlinear operators in reflexive Banach spaces, Numer. Funct. Anal. Optim. 24, 489-508 (2003)

5. Censor, Y., Reich, S., Iterations of paracontractions and firmly nonexpansive operators with applications to feasibility and optimization, $O p$ timization 37, 323-339 (1996)

6. Combettes, P. L., Hirstoaga, S. A., Equilibrium programming in Hilbert spaces, J. Nonlinear Convex Anal. 6, 117-136 (2005)

7. Kamimura, s., Takahashi, W., Strong convergence of a proximal-type algorithm in a Banach space, SIAM J. Optim. 13, 938-945 (2002)

8. Matsushita, S., Takahashi, W., A strong convergence theorem for relatively nonexpansive mappings in Banach spaces, J. Approx. Theor. 134, 257-266 (2005) 
9. Nilsrakoo, W., Saejung, S., Strong convergence to common fixed points of countable relatively quasi-nonexpansive mappings, Fixed Point Theory and Applications. Article ID 312454, 19 pages, doi:10.1155/2008/312454(2008)

10. Qin, X., Cho, Y. J., Kang, S. M., Convergence theorems of common elements for equilibrium problems and fixed point problems in Banach spaces, J. Comput. Appl. Math. 225, 20-30 (2009)

11. Takahashi, W., Zembayashi, K., Strong and weak convergence theorems for equilibrium problems and relatively nonexpansive mappings in Banach spaces, Nonlinear Anal. 70, 45-57 (2009)

12. Takahashi, W., Zembayashi, K., Strong convergence theorems by a new iteration method for equilibrium problems and relatively nonexpansive mappings. Fixed Point Theory and Applications. Article ID 528476, p. 11 (2008)

13. Yekini, S., Iterative approximation method for finite family of relatively quasi nonexpansive mappings and systems of equilibrium problems, $J$. Glob. Optim. Article ID , doi 10,1007/s10898-010-9619-4 (2010)

14. Xu, H. K., Inequalities in Banach spaces with applications, Nonlinear Anal. 16 (2), 1127-1138 (1991)

Received: April 15, 2014 